\title{
What Orchestrates the Self-Assembly of Glycine Molecules on $\mathrm{Cu}(100)$ ?
}

\author{
Ken Kanazawa, Atsushi Taninaka, Osamu Takeuchi, and Hidemi Shigekawa* \\ Institute of Applied Physics, CREST-JST, University of Tsukuba, Tsukuba 305-8573 Japan
}

(Received 18 June 2007; published 20 November 2007)

\begin{abstract}
The structures of two competing phases and their interrelationship in the self-organization of glycine molecules on a $\mathrm{Cu}(100)$ surface were clarified. Despite their similar structural energies predicted using first-principles calculation, completely different mechanisms were found to stabilize the two phases. The balance and coordination of the two mechanisms that induce a variety of self-assembled structures in this attractive system were revealed. Furthermore, the importance of the microscopic arrangement of the molecules in designing the macroscopic electronic structures was directly demonstrated.
\end{abstract}

PACS numbers: 81.16.Dn, 68.37.Ef

The formation of self-assembled structures is a result of the balance of interactions among materials and environmental conditions, which produces a variety in the macroscopic functions of the system. Recent developments in the formation of nanostructures have been achieved by controlling the interactions depending, for example, on the electronic and conformational structures, strains, and chemical reactions [1-3]. However, the balance of these interactions is, in general, extremely delicate and complex, and furthermore, some interactions are hidden behind the dynamics apparently observed. In a solution, for example, the structure of the solvents plays an important role through the solvophobic effects on the nanostructural formation of solute materials [4]. Even in the case of a more solid environment such as adsorbate molecules on a substrate material, reconstruction of the substrate may be induced to produce a stable superstructure [5], which may be hidden behind the apparent molecular arrangement. Time-evolutional change is also included in the interactions depending on the growth process [6]. Since self-organization is governed by the interactions of these fine variations, which determine the macroscopic functions, evaluation of the real dynamics is of great importance for further understanding, and subsequently the manipulation of the mechanism.

Here, we demonstrate the intriguing two-step mechanism that governs the self-assembly of polarized molecules with chirality on a transition metal, glycine molecules on a $\mathrm{Cu}(100)$ surface. The structures of two competing phases and their interrelationship that induces a variety of selfassembled structures in this attractive system were revealed. Furthermore, the characteristic relationship between the microscopic molecular arrangements and the macroscopic electronic structure is discussed.

The glycine molecule is the simplest amino acid with carboxyl and amino groups, which are both common for all amino acids, and is one of the fundamental components of biological molecules. Owing to the basic and practical interest in this material, adsorption of this molecule on a $\mathrm{Cu}$ surface has been widely studied [3,7-9]. The observation of the anisotropic two-dimensional (2D) free- electronlike electronic structure has made this system more attractive [3]. Among all amino acids, glycine is the only molecule that does not have chirality, but enantiomeric isomers appear on the $\mathrm{Cu}$ surface, through the dissociation of hydrogen, depending on the directional relationship of the two groups in the adsorbed form as schematically illustrated in Fig. 1(a) [7]. The adsorption properties of this system have been expected to provide us with the basis for understanding, and a method of applying, the mechanism of the self-assembly of a polarized molecule with chirality.

Despite the extensive studies, however, there still remains controversy even in determining the ground state of this system, namely, the $c(2 \times 4)$ or $p(2 \times 4)$ structure [Figs. 1(b) and 1(c)]. Recent theoretical calculation has shown that the $c(2 \times 4)$ and $p(2 \times 4)$ structures have similar structural energies [9]. In fact, from scanning tunneling microscopy (STM) observation at room temperature (RT) it was found, for example, that although molecules were unstable at low densities, two structures with the $c(2 \times 4)$ or $p(2 \times 4)$ periodicity coexisted for high deposition concentrations [8]. In contrast, only the $p(2 \times 4)$ pattern was observed using low energy electron diffraction (LEED) at RT, and formation of the $p(2 \times 4)$ structure was confirmed using STM at $5 \mathrm{~K}$, indicating the selective growth of one phase $[3,7]$. These results suggest the existence of some complex mechanism behind the selfassembly of this system. (a)

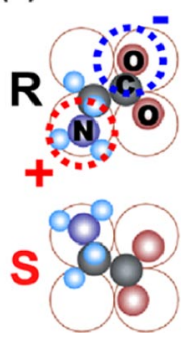

(b) $p(2 \times 4)$ heterochiral

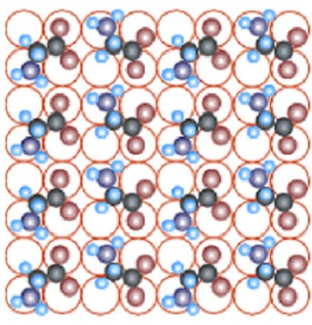

(c) $c(2 \times 4)$ homochiral

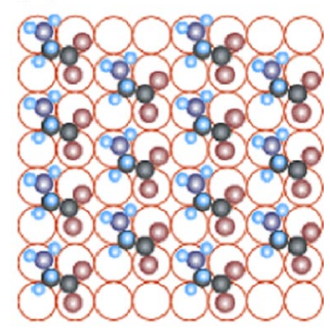

FIG. 1 (color online). (a) Two conformations of an adsorbed glycine molecule, and molecular arrangements of (b) $p(2 \times 4)$ and (c) $c(2 \times 4)$ structures. 
In response to the fundamental and intriguing requirement of clarifying the mechanism that governs the selfassembly of this basic and practically important material, we performed a detailed analysis on the growth of this system using scanning tunneling microscopy or spectroscopy (STM/STS).

The sample preparation [3] and STM measurements were performed under ultrahigh-vacuum conditions (base pressure $<1 \times 10^{-8} \mathrm{~Pa}$ ). An electrochemically sharpened tungsten tip $(\phi=0.3 \mathrm{~mm})$ was used.

Figure 2 shows STM images obtained at $5 \mathrm{~K}$ for bare and glycine-deposited $\mathrm{Cu}(100)$ surfaces. For an increase in the concentration of glycine molecules the domains consisting of the $p(2 \times 4)$ arrangement grew, which appears to be
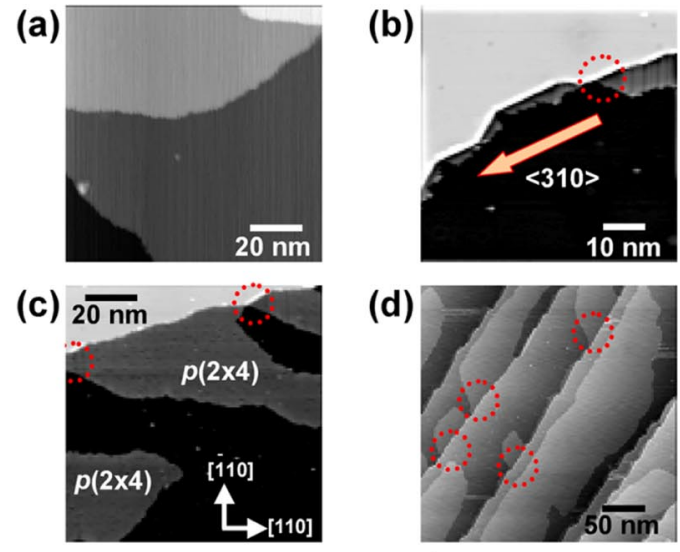

(d)
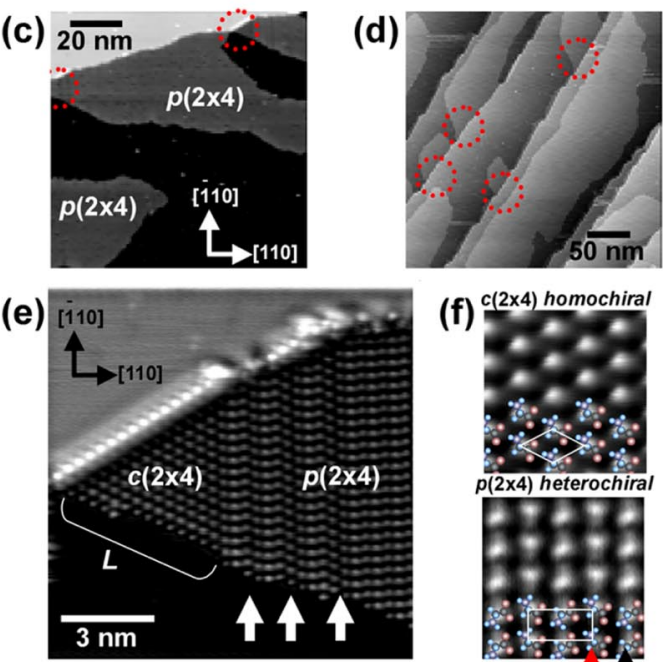

(g)

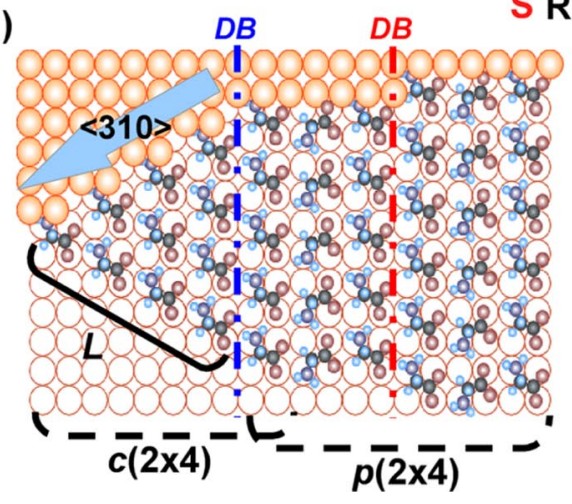

FIG. 2 (color online). STM images obtained at $5 \mathrm{~K}$ for the bare (a) and glycine-deposited $\mathrm{Cu}(100)$ surfaces ((b) 0.2, (c) 0.4, and (d) $0.6 \mathrm{ML}$ ). (e) A magnified STM image of the area indicated by red circles in (b) $-(d)$. (f) Magnifications of $c(2 \times 4)$ and $p(2 \times$ 4) areas with molecular arrangements, (g) Structural models of the molecular arrangements in (e). consistent with the recent STM/LEED results [3,7]. However, the details are more complicated.

Noteworthy points, observed for the fist time, are as follows: first, the formation of $\langle 310\rangle$ steps, which never appear on the bare $\mathrm{Cu}(100)$ surface, was observed. Second, there are domains that exhibit a triangular shape at the edge of the $p(2 \times 4)$ domains as indicated by red circles in Figs. 2(b)-2(d). Figure 2(e) shows a magnified STM image of such an area. From the STM image together with the theoretical result [9], the phase of the triangular shape is determined to be of the $c(2 \times 4)$ homochiral structure [Figs. 1(c) and 2(f)], the existence of which has been an object of long-term controversy [7,8]. The $c(2 \times 4)$ domain always grows from a $\langle 310\rangle$ step on the lower terrace. A molecular row was observed on the upper side of the $\langle 310\rangle$ step edge where the $c(2 \times 4)$ structure is on the lower terrace. Third, all $p(2 \times 4)$ domains are formed in the vicinity of the $c(2 \times 4)$ triangular island and the edge of the step that differs from the $\langle 310\rangle$ step. In contrast to the case of the $c(2 \times 4)$ structure formed at the $\langle 310\rangle$ steps, no molecular rows were observed on the upper of the step edge. Fourth, there are boundaries in the $p(2 \times 4)$ area as indicated by the white arrows in Fig. 2(e), where molecular rows have the $c(2 \times 4)$ arrangement. On the terrace, far from the $\langle 310\rangle$ steps [for example, Fig. 2(c)], a complete $p(2 \times 4)$ arrangement without $c(2 \times 4)$ boundaries is formed. In other words, a $c(2 \times 4)$ arrangement is only formed when it is in contact with the $\langle 310\rangle$ step edge.

Figure 2(g) is an illustration of the structural models of the molecular arrangements. The molecular arrangement of the $c(2 \times 4)$ structure and its positional relationship with the $\langle 310\rangle$ step were determined by comparing the STM image with theoretical calculations [Fig. 2(f)] [9]. This structure can be supported as follows: as shown in Fig. 1(a), amino and carboxyl groups in an adsorbed glycine molecule are positively and negatively charged, respectively. On a metal surface, the upper (lower) side of a step is positively (negatively) polarized according to the well-known Smoluchowski effect [10]. Therefore, glycine molecules are adsorbed onto the surface with the positively charged amino groups in contact with the lower edge of the $\langle 310\rangle$ step, which is negatively charged. Then the subsequent glycine molecules form the $c(2 \times 4)$ structure as a result of the attractive interaction between the oxygen $(\mathrm{O})$ atoms in the molecules adsorbed ahead and the nitrogen $(\mathrm{N})$ atoms in the subsequent molecules.

Why is the $c(2 \times 4)$ domain always a triangular shape, even though the $\mathrm{Cu}(100)$ surface has a fourfold rotational symmetry? Since the edge indicated by $L$ in Figs. 2(e) and $2(\mathrm{~g})$ is always straight and no partially added molecules were observed, the interaction between the edge and the additional molecule, based on the attractive interaction between $\mathrm{N}$ and $\mathrm{O}$ atoms, is not strong enough to stabilize the structure. Positive and negative parts are arranged alternately along the edge $L$, which may reduce the interaction of this edge with the additional molecules. 
Since the upper side of a step is positively polarized, the molecular arrangement of the $c(2 \times 4)$ structure on the upper side of the step is supposed to have an orientation similar to that on the lower terrace. On the upper $\langle 310\rangle$ step edge, the growth of the $c(2 \times 4)$ structure was slow, indicating the weaker interaction in this direction. No negatively charged $\mathrm{O}$ atoms on its island edge and the formation of an intramolecular hydrogen bond (N-H-O) [11] may cause a weaker molecular interaction resulting in the observed slow growth in this direction.

Considering the molecular arrangement of the $c(2 \times 4)$ structure with the fact that the $p(2 \times 4)$ domain exists only on one side of the $c(2 \times 4)$ island, that is in the vicinity of the edge along the [ $\overline{1} 10]$ direction [Fig. 2(e)], the molecular orientation in the $p(2 \times 4)$ domain was determined as illustrated in Fig. 2(g). In both structures, molecules are well ordered along the [110] direction to form a molecular row. As was pointed out, there are boundaries formed by the $c(2 \times 4)$ structure in the $p(2 \times 4)$ area as indicated by the white arrows in Fig. 2(e) [red dashed-dotted line in Fig. 2(g)]. Since a phase defect is induced by the substitution of a molecular row of one chirality with a molecular row of the other chirality [appearance of SS or RR ordering instead of the alternate SR ordering as in Fig. 2(f)], the molecular interaction in each row is stronger than that between the rows. In fact, tenfold-different anisotropy in the effective masses was observed for the 2D electronic structure formed in the $p(2 \times 4)$ structure [3], indicating a larger overlap of electronic states in the molecules along the [110] direction, which results in a stronger interaction.

Which is the ground state of this system? One important point is that the step edge forming the $c(2 \times 4)$ structural domain boundaries is a short $\langle 310\rangle$ step. Thus, the relationship between the $c(2 \times 4)$ structure and the $\langle 310\rangle$ step, that is the formation of the $\langle 310\rangle$ step through molecular adsorption, is considered to play an essential role in the self-organization of this system.

To investigate the $\langle 310\rangle$ step formation process and its stability in relation to the $c(2 \times 4)$ structure, we performed a continuous observation of one location at RT. Figures 3(a) and 3(c)-3(e) show a sequence of STM images obtained every $512 \mathrm{sec}$. Figure 3(b) is a magnification of the area indicated by the arrow $T$ in Fig. 3(a). A remarkable point is that straight $\langle 310\rangle$ steps are formed and, as shown in the lowest wide terrace, molecules are adsorbed along the straight $\langle 310\rangle$ steps, and stable triangular $c(2 \times 4)$ islands exist at the short step area even at RT; although some steps exhibit fluctuations. A $c(2 \times 4)$ phase with $\langle 310\rangle$ steps was observed on the narrow terrace, where the width is close to the size of the $c(2 \times 4)$ triangle (10-15 molecular edge size at RT). The $c(2 \times 4)$ structural area along the straight $\langle 310\rangle$ steps is darker (lower) than the terrace far from the steps, indicating that the wide terrace area is not a bare $\mathrm{Cu}$ surface, but many molecules diffuse on the surface as pointed out in a previous paper [8]. Despite this, no $p(2 \times 4)$ island formation was observed.
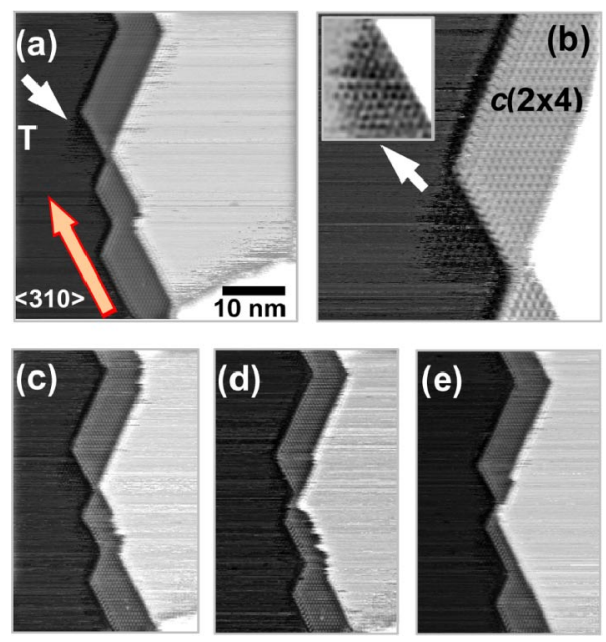

FIG. 3 (color online). (a), (c)-(e) show a sequence of STM images obtained every $512 \mathrm{sec}$; (b) shows a magnification of the area indicated by the arrow $T$ in (a). The inset in (b) is a highcontrast image of the triangular structure.

These results suggest that the $c(2 \times 4)$ structure is more stable. When the temperature is lowered, the surface is expected to be covered by the $c(2 \times 4)$ phase. However, in the terrace far from the $\langle 310\rangle$ steps [see Fig. 2(c)], a complete $p(2 \times 4)$ arrangement without $c(2 \times 4)$ boundaries is formed. A $c(2 \times 4)$ arrangement is not formed without the molecular rows being in contact with the $\langle 310\rangle$ step edge. Therefore, although a $c(2 \times 4)$ triangular island is necessary as a nucleus to stabilize the $p(2 \times 4)$ island, the $p(2 \times 4)$ arrangement appears to be more stable than the $c(2 \times 4)$ arrangement at low temperatures.

The results obtained at $5 \mathrm{~K}$ and RT indicate the existence of the two different mechanisms that govern the observed self-assembly of glycine molecules. When glycine molecules are deposited onto a $\mathrm{Cu}(100)$ surface at $\mathrm{RT},\langle 310\rangle$ steps, which are never observed on the bare surface, are formed by the adsorption of the molecules, and $c(2 \times 4)$ structure islands grow from the steps until a triangular shape with the edge corresponding to the stable length depending on the temperature is achieved.

Despite the strong interaction at the $\langle 310\rangle$ steps, which stabilizes the $c(2 \times 4)$ phase, as the temperature is lowered, $p(2 \times 4)$ islands grow from the edge of the $c(2 \times 4)$ triangle along the [110] direction [blue dashed-dotted line in Fig. 2(g)]. When the sample temperature is maintained at RT for a long enough time to form a narrow terrace sandwiched by the $\langle 310\rangle$ steps, the $c(2 \times 4)$ phase is stabilized there; however, the $c(2 \times 4)$ phase does not cover the entire surface. This change indicates that the molecules in the $p(2 \times 4)$ arrangement have a stronger interaction with the substrate at lower temperatures. Recent STM work has revealed the formation of a $2 \mathrm{D}$ electronic structure for the $p(2 \times 4)$ structure [3]. Does this make the $p(2 \times 4)$ structure more stable than $c(2 \times 4)$ ? To investigate the temperature dependence of the electronic structure, we performed STS at RT and $5 \mathrm{~K}$ on an area that 

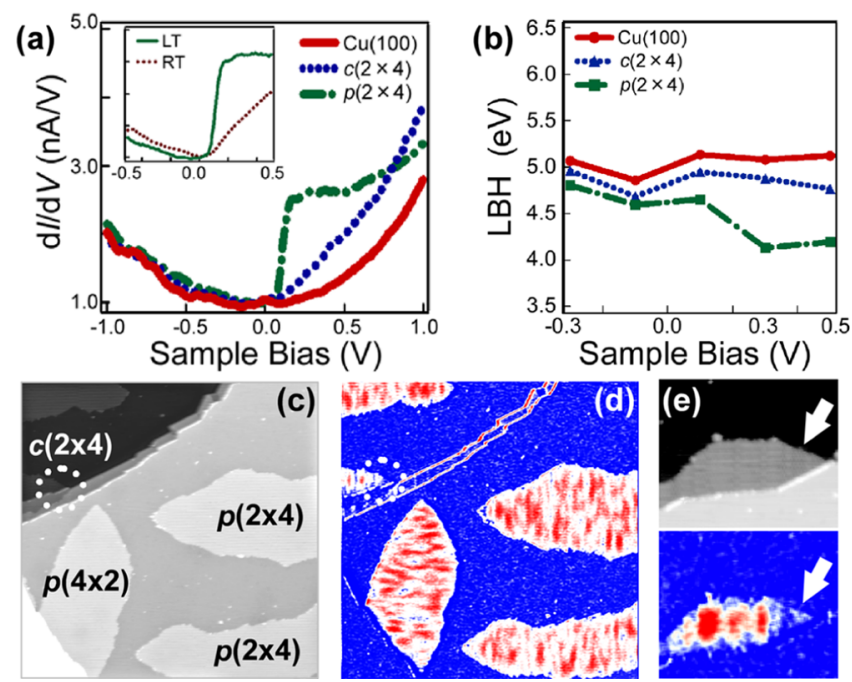

FIG. 4 (color online). (a) Spectra obtained at 5 K. (b) $\mathrm{LBH}$ spectra obtained at $5 \mathrm{~K}$ (set point: $1 \mathrm{M} \Omega, 0.1 \mathrm{nA}, 0.1 \mathrm{~V}$ ). Data were obtained by measuring the $I-Z$ curve as the STM tip was retracted $0.3 \mathrm{~nm}$ from the set point; (c), (d) show topographic and $d I / d V$ images of a glycine-deposited surface $(200 \mathrm{~nm} \times$ $200 \mathrm{~nm},+0.11 \mathrm{~V}, 1.2 \mathrm{nA}$ ), and (e) shows magnified topographic and $d I / d V$ images, where $c(2 \times 4)$ triangular (indicated by white arrow) and neighboring $p(2 \times 4)$ structures coexist $(+0.13 \mathrm{~V}, 0.2 \mathrm{nA})$.

includes a bare $\mathrm{Cu}$ substrate, and $c(2 \times 4)$ and $p(2 \times 4)$ structures. Since molecules are unstable at RT, we prepared a sample with a high deposition concentration to stabilize the structures for the measurement at RT.

Figure 4(a) shows the spectra obtained at $5 \mathrm{~K}$ [12]. A sharp edge of the local density of states near $+100 \mathrm{mV}$, which corresponds to the anisotropic $2 \mathrm{D}$ electronic structure formed in the $p(2 \times 4)$ structure [3], does not appear for the $c(2 \times 4)$ structure. Figures 4(c) and 4(d) show STM and $d I / d V$ images (lock-in modulation: $8 \mathrm{mV}, 2.74 \mathrm{kHz}$ ) of a glycine-deposited surface, and Fig. 4(e) shows magnified images where the $c(2 \times 4)$ triangular and $p(2 \times 4)$ phases coexist. As expected, the standing wave that appears in the $p(2 \times 4)$ structure is not observed in the $c(2 \times$ 4) structure. As shown in the inset of Fig. 4(a), the sharp edge disappears at RT, and the spectrum for the $p(2 \times 4)$ structure becomes similar to that of the $c(2 \times 4)$.

These results suggest that the electronic state observed for the $p(2 \times 4)$ structure at $5 \mathrm{~K}$ is a result of the interaction between the molecular layer and the $\mathrm{Cu}(100)$ substrate [3]. To confirm this point, we performed local barrier height (LBH) measurement by STM for the bare Cu substrate and $c(2 \times 4)$ and $p(2 \times 4)$ structures at $5 \mathrm{~K}$; Fig. 4(b) shows the result. Although there is an effect of the 2D electronic structure for the region of bias voltage larger than $0.1 \mathrm{~V}$, the value of LBH obtained for the $p(2 \times 4)$ structure is the smallest over the entire voltage range, suggesting a larger charge transfer from the molecular layer to the $\mathrm{Cu}$ substrate in the $p(2 \times 4)$ structure. This is due to the coupling of the electronic states between the molecular layer and the $\mathrm{Cu}$ substrate. This interaction must stabilize the $p(2 \times 4)$ structure, making the $p(2 \times 4)$ phase the ground state of this system. In fact, as observed in the change in the STS spectra shown in Fig. 4(a), the interaction breaks off and the stronger interaction through the formation of the $\langle 310\rangle$ step is dominant at RT, resulting in the apparent high stability of the $c(2 \times 4)$ arrangement. Despite the similar structural energies predicted using firstprinciples calculation, microscopic molecular arrangement of glycine molecules on a $\mathrm{Cu}$ surface produces characteristic variations in the associated interactions and macroscopic electronic structures.

In conclusion, the structures of two competing phases, $c(2 \times 4)$ and $p(2 \times 4)$, and their interrelationship that governs the observed variety in the self-organization of glycine molecules on a $\mathrm{Cu}(100)$ surface, which had been a long-term controversy, were clarified for the first time. The $c(2 \times 4)$ phase was produced by the formation of $\langle 310\rangle$ steps, which has not been observed on the bare $\mathrm{Cu}(100)$ surface, through the adsorption of glycine molecules. Direct observations of the formation and fluctuation of the $\langle 310\rangle$ step at RT indicate a strong interaction between glycine molecules for the $c(2 \times 4)$ phase and the $\langle 310\rangle$ step. The hidden role of the $c(2 \times 4)$ structure as a nucleus for the growth of the $p(2 \times 4)$ structure was also revealed. Finally, we characterized the difference in the electronic structures between the two arrangements, which is the origin of the mechanism that stabilizes the $p(2 \times 4)$ structure as the ground state of the system. These results are direct evidence of the important role of local molecular arrangement in the design of the macroscopic electronic structure.

We thank Ms. Rie Yamashita, in our group at University of Tsukuba, for her help in preparing this Letter.

*http://dora.ims.tsukuba.ac.jp/

[1] J. V. Barth, G. Costantini, and K. Kern, Nature (London) 437, 678 (2005).

[2] G. Pawin et al., Science 313, 961 (2006).

[3] K. Kanazawa et al., J. Am. Chem. Soc. 129, 740 (2007), and references therein.

[4] S. Yasuda, I. Suzuki, K. Shinohara, and H. Shigekawa, Phys. Rev. Lett. 96, 228303 (2006).

[5] A. Kuhnle et al., Phys. Rev. Lett. 93, 086101 (2004).

[6] J. Wintterlin et al., Science 278, 1931 (1997).

[7] V. Efstathiou and D.P. Woodruff, Surf. Sci. 531, 304 (2003).

[8] X. Zhao et al., Mater. Sci. Eng., C 16, 41 (2001).

[9] K. Mae and Y. Morikawa, Surf. Sci. 553, L63 (2004).

[10] R. Smoluchowski, Phys. Rev. 60, 661 (1941).

[11] L. R. Santiago et al., J. Phys. Chem. A 104, 1256 (2000).

[12] We adopted $d I / d V$ in Fig. 4(a) instead of $(d I / d V) /(I / V)$ to remove the artificial effect by the singularity at zero bias voltage and to reduce the influence of the rapid increase in I on the spectra. C. Wagner, R. Franke, and T. Fritz, Phys. Rev. B 75, 235432 (2007). 